\title{
Automatic annotation of operons responsible for O-antigen synthesis
}

\author{
Danil Zilov \\ Laboratory of Applied Genomics, \\ SCAMT, ITMO University, St. \\ Petersburg, Russia \\ zilov@scamt-itmo.ru
}

\author{
Polina Chesnokova \\ Laboratory of Applied Genomics, \\ SCAMT, ITMO University, St. \\ Petersburg, Russia \\ chesnokova@scamt-itmo.ru
}

\author{
Alexey Komissarov \\ Laboratory of Applied Genomics, \\ SCAMT, ITMO University, St. \\ Petersburg, Russia \\ komissarov@scamt-itmo.ru
}

\begin{abstract}
Unfortunately, the most ready-made tools for serotype determination are limited to genome $E$. Coli only. However, there is a problem due to the low accuracy of $\mathrm{O}$-antigen cluster identification in the genome. Pipeline, which was created due the work, solves this problem and can be used to find and annotate a cluster of genes in genome of the most gram-negative bacteria.
\end{abstract}

Keywords - O-antigen, genome assembly, annotation of genome, pipeline

\section{Motivation and aim}

\section{Motivation}

$\mathrm{O}$-antigen is a polysaccharide, the major component of the outer bacterial membrane. O-antigen biosynthesis is encoded by genes, which are often clustered. Despite the simplicity of the cluster structure, its identification in microbial DNA is still a problem.

It is well known, that in the structure of O-antigen there are specific sites of binding to antibodies of the host organism, the interaction with which leads to the emergence of an immune response to infection. We are interested in the way these sites are organized. Unfortunately, the most readymade tools for serotype determination are limited to genome E. Coli only. Therefore, we started to build our own tool.

Aim

Our goal is to create a pipeline that will automate the search, assembly, filtration and annotation of a cluster of genes responsible for the synthesis of $\mathrm{O}$-antigen, for most gram-negative bacteria.

\section{Methods}

We used Python programming language, Snakemake workflow management system, BLAST and NCBI databases
Conda package manager and few custom scripts to create pipeline.

To run the pipeline raw sequence reads or assembled genome is needed.

The main stages of data processing:

- Assessing the quality of input data, removing adapters and optical duplicates;

- Genome assembly using SPAdes and Unicycler;

- Checking for contamination and plasmids;

- Removal of contaminants and plasmids, if any;

- Annotation by Prokka and eggNOG;

- Functional annotation, extraction of genes involved in $\mathrm{O}$ antigen biosynthesis;

- Abstract of operons containing genes involved in Oantigen biosynthesis taking into account possible assembly errors and contigs breaks.

We are currently annotating $\mathrm{O}$-antigens of all gramnegative bacteria available in the NCBI database. The resulting information will be used to improve the existing Pipeline.

\section{Results}

The created pipeline significantly saves time for serotyping gram-negative bacteria. Additionally, it can be used in the detection of outer membrane components related to both virulence of microorganisms and symbiotic interaction between bacteria and plants. 